\title{
Limit on the $B^{0} \rightarrow \rho^{0} \rho^{0}$ Branching Fraction and Implications for the Cabibbo-Kobayashi-Maskawa Angle $\alpha$
}

B. Aubert, ${ }^{1}$ R. Barate, ${ }^{1}$ D. Boutigny, ${ }^{1}$ F. Couderc,${ }^{1}$ Y. Karyotakis,${ }^{1}$ J. P. Lees,${ }^{1}$ V. Poireau, ${ }^{1}$ V. Tisserand, ${ }^{1}$ A. Zghiche,${ }^{1}$ E. Grauges-Pous, ${ }^{2}$ A. Palano, ${ }^{3}$ A. Pompili, ${ }^{3}$ J. C. Chen, ${ }^{4}$ N. D. Qi ${ }^{4}$ G. Rong, ${ }^{4}$ P. Wang, ${ }^{4}$ Y. S. Zhu, ${ }^{4}$ G. Eigen, ${ }^{5}$ I. Ofte, ${ }^{5}$ B. Stugu, ${ }^{5}$ G. S. Abrams, ${ }^{6}$ A. W. Borgland, ${ }^{6}$ A. B. Breon, ${ }^{6}$ D. N. Brown, ${ }^{6}$ J. Button-Shafer, ${ }^{6}$ R. N. Cahn, ${ }^{6}$ E. Charles, ${ }^{6}$ C. T. Day, ${ }^{6}$ M. S. Gill, ${ }^{6}$ A. V. Gritsan, ${ }^{6}$ Y. Groysman, ${ }^{6}$ R. G. Jacobsen, ${ }^{6}$ R. W. Kadel, ${ }^{6}$ J. Kadyk, ${ }^{6}$ L. T. Kerth, ${ }^{6}$ Yu. G. Kolomensky, ${ }^{6}$ G. Kukartsev ${ }^{6}{ }^{\text {G. }}$ Lynch, ${ }^{6}$ L. M. Mir, ${ }^{6}$ P. J. Oddone, ${ }^{6}$ T. J. Orimoto, ${ }^{6}$ M. Pripstein, ${ }^{6}$ N. A. Roe, ${ }^{6}$ M. T. Ronan, ${ }^{6}$ W. A. Wenzel, ${ }^{6}$ M. Barrett, ${ }^{7}$ K. E. Ford, ${ }^{7}$ T. J. Harrison, ${ }^{7}$ A. J. Hart, ${ }^{7}$ C. M. Hawkes, ${ }^{7}$ S. E. Morgan, ${ }^{7}$ A. T. Watson, ${ }^{7}$ M. Fritsch, ${ }^{8}$ K. Goetzen,${ }^{8}$ T. Held,${ }^{8}$ H. Koch,${ }^{8}$ B. Lewandowski, ${ }^{8}$ M. Pelizaeus, ${ }^{8}$ K. Peters, ${ }^{8}$ T. Schroeder, ${ }^{8}$ M. Steinke, ${ }^{8}$ J. T. Boyd,${ }^{9}$ J. P. Burke, ${ }^{9}$ N. Chevalier, ${ }^{9}$ W. N. Cottingham, ${ }^{9}$ M. P. Kelly, ${ }^{9}$ T. E. Latham, ${ }^{9}$ F. F. Wilson, ${ }^{9}$ T. Cuhadar-Donszelmann, ${ }^{10}$ C. Hearty,${ }^{10}$ N. S. Knecht, ${ }^{10}$ T. S. Mattison, ${ }^{10}$ J. A. McKenna, ${ }^{10}$ D. Thiessen, ${ }^{10}$ A. Khan ${ }^{11}$ P. Kyberd, ${ }^{11}$ L. Teodorescu, ${ }^{11}$ A. E. Blinov, ${ }^{12}$ V.E. Blinov, ${ }^{12}$ V. P. Druzhinin, ${ }^{12}$ V. B. Golubev, ${ }^{12}$ V. N. Ivanchenko, ${ }^{12}$ E. A. Kravchenko, ${ }^{12}$ A. P. Onuchin, ${ }^{12}$ S. I. Serednyakov, ${ }^{12}$ Yu. I. Skovpen, ${ }^{12}$ E. P. Solodov, ${ }^{12}$ A. N. Yushkov, ${ }^{12}$ D. Best, ${ }^{13}$ M. Bruinsma, ${ }^{13}$ M. Chao, ${ }^{13}$ I. Eschrich, ${ }^{13}$ D. Kirkby, ${ }^{13}$ A. J. Lankford, ${ }^{13}$ M. Mandelkern, ${ }^{13}$ R. K. Mommsen, ${ }^{13}$ W. Roethel, ${ }^{13}$ D. P. Stoker, ${ }^{13}$ C. Buchanan, ${ }^{14}$ B. L. Hartfiel, ${ }^{14}$ A. J. R. Weinstein, ${ }^{14}$ S. D. Foulkes, ${ }^{15}$ J. W. Gary, ${ }^{15}$ O. Long, ${ }^{15}$ B. C. Shen, ${ }^{15}$ K. Wang, ${ }^{15}$ D. del Re,${ }^{16}$ H. K. Hadavand, ${ }^{16}$ E. J. Hill, ${ }^{16}$ D. B. MacFarlane, ${ }^{16}$ H. P. Paar, ${ }^{16}$ Sh. Rahatlou, ${ }^{16}$ V. Sharma, ${ }^{16}$ J. W. Berryhill, ${ }^{17}$ C. Campagnari, ${ }^{17}$ A. Cunha, ${ }^{17}$ B. Dahmes,,${ }^{17}$ T. M. Hong, ${ }^{17}$ A. Lu, ${ }^{17}$ M. A. Mazur, ${ }^{17}$

J. D. Richman, ${ }^{17}$ W. Verkerke, ${ }^{17}$ T. W. Beck, ${ }^{18}$ A. M. Eisner, ${ }^{18}$ C. J. Flacco, ${ }^{18}$ C. A. Heusch, ${ }^{18}$ J. Kroseberg, ${ }^{18}$

W. S. Lockman, ${ }^{18}$ G. Nesom, ${ }^{18}$ T. Schalk,${ }^{18}$ B. A. Schumm, ${ }^{18}$ A. Seiden, ${ }^{18}$ P. Spradlin, ${ }^{18}$ D. C. Williams, ${ }^{18}$ M. G. Wilson, ${ }^{18}$ J. Albert, ${ }^{19}$ E. Chen, ${ }^{19}$ G. P. Dubois-Felsmann, ${ }^{19}$ A. Dvoretskii, ${ }^{19}$ D. G. Hitlin, ${ }^{19}$ I. Narsky, ${ }^{19}$ T. Piatenko, ${ }^{19}$ F. C. Porter, ${ }^{19}$ A. Ryd,${ }^{19}$ A. Samuel, ${ }^{19}$ S. Yang, ${ }^{19}$ S. Jayatilleke, ${ }^{20}$ G. Mancinelli, ${ }^{20}$ B. T. Meadows,${ }^{20}$ M. D. Sokoloff, ${ }^{20}$ F. Blanc,${ }^{21}$

P. Bloom, ${ }^{21}$ S. Chen, ${ }^{21}$ W. T. Ford, ${ }^{21}$ U. Nauenberg, ${ }^{21}$ A. Olivas, ${ }^{21}$ P. Rankin,${ }^{21}$ W. O. Ruddick, ${ }^{21}$ J. G. Smith,${ }^{21}$ K. A. Ulmer ${ }^{21}$ J. Zhang, ${ }^{21}$ L. Zhang, ${ }^{21}$ A. Chen, ${ }^{22}$ E. A. Eckhart, ${ }^{22}$ J. L. Harton, ${ }^{22}$ A. Soffer, ${ }^{22}$ W. H. Toki, ${ }^{22}$ R. J. Wilson, ${ }^{22}$ Q. Zeng, ${ }^{22}$ B. Spaan,${ }^{23}$ D. Altenburg, ${ }^{24}$ T. Brandt, ${ }^{24}$ J. Brose,${ }^{24}$ M. Dickopp,${ }^{24}$ E. Feltresi, ${ }^{24}$ A. Hauke, ${ }^{24}$ H. M. Lacker,${ }^{24}$

E. Maly, ${ }^{24}$ R. Nogowski, ${ }^{24}$ S. Otto, ${ }^{24}$ A. Petzold, ${ }^{24}$ G. Schott, ${ }^{24}$ J. Schubert, ${ }^{24}$ K. R. Schubert, ${ }^{24}$ R. Schwierz, ${ }^{24}$

J. E. Sundermann, ${ }^{24}$ D. Bernard ${ }^{25}$ G. R. Bonneaud,${ }^{25}$ P. Grenier, ${ }^{25}$ S. Schrenk,${ }^{25}$ Ch. Thiebaux,${ }^{25}$ G. Vasileiadis, ${ }^{25}$ M. Verderi, ${ }^{25}$ D. J. Bard ${ }^{26}$ P. J. Clark, ${ }^{26}$ F. Muheim, ${ }^{26}$ S. Playfer, ${ }^{26}$ Y. Xie,${ }^{26}$ M. Andreotti, ${ }^{27}$ V. Azzolini, ${ }^{27}$ D. Bettoni, ${ }^{27}$ C. Bozzi, ${ }^{27}$ R. Calabrese,${ }^{27}$ G. Cibinetto, ${ }^{27}$ E. Luppi, ${ }^{27}$ M. Negrini,${ }^{27}$ L. Piemontese, ${ }^{27}$ A. Sarti, ${ }^{27}$ F. Anulli, ${ }^{28}$

R. Baldini-Ferroli, ${ }^{28}$ A. Calcaterra, ${ }^{28}$ R. de Sangro, ${ }^{28}$ G. Finocchiaro, ${ }^{28}$ P. Patteri, ${ }^{28}$ I. M. Peruzzi, ${ }^{28}$ M. Piccolo, ${ }^{28}$ A. Zallo, ${ }^{28}$ A. Buzzo, ${ }^{29}$ R. Capra ${ }^{29}$ R. Contri, ${ }^{29}$ G. Crosetti, ${ }^{29}$ M. Lo Vetere, ${ }^{29}$ M. Macri, ${ }^{29}$ M. R. Monge, ${ }^{29}$ S. Passaggio, ${ }^{29}$

C. Patrignani, ${ }^{29}$ E. Robutti ${ }^{29}$ A. Santroni, ${ }^{29}$ S. Tosi,${ }^{29}$ S. Bailey, ${ }^{30}$ G. Brandenburg, ${ }^{30}$ K. S. Chaisanguanthum, ${ }^{30}$ M. Morii, ${ }^{30}$ E. Won, ${ }^{30}$ R. S. Dubitzky, ${ }^{31}$ U. Langenegger, ${ }^{31}$ J. Marks, ${ }^{31}$ U. Uwer, ${ }^{31}$ W. Bhimji, ${ }^{32}$ D. A. Bowerman, ${ }^{32}$

P. D. Dauncey, ${ }^{32}$ U. Egede, ${ }^{32}$ J. R. Gaillard, ${ }^{32}$ G. W. Morton, ${ }^{32}$ J. A. Nash, ${ }^{32}$ M. B. Nikolich, ${ }^{32}$ G. P. Taylor, ${ }^{32}$

M. J. Charles, ${ }^{33}$ G. J. Grenier, ${ }^{33}$ U. Mallik, ${ }^{33}$ A. K. Mohapatra, ${ }^{33}$ J. Cochran, ${ }^{34}$ H. B. Crawley, ${ }^{34}$ J. Lamsa, ${ }^{34}$ W. T. Meyer, ${ }^{34}$ S. Prell, ${ }^{34}$ E. I. Rosenberg, ${ }^{34}$ A. E. Rubin, ${ }^{34}$ J. Yi, ${ }^{34}$ N. Arnaud, ${ }^{35}$ M. Davier, ${ }^{35}$ X. Giroux, ${ }^{35}$ G. Grosdidier,${ }^{35}$ A. Höcker, ${ }^{35}$ F. Le Diberder, ${ }^{35}$ V. Lepeltier, ${ }^{35}$ A. M. Lutz, ${ }^{35}$ T. C. Petersen, ${ }^{35}$ M. Pierini, ${ }^{35}$ S. Plaszczynski, ${ }^{35}$ M. H. Schune, ${ }^{35}$

G. Wormser, ${ }^{35}$ C. H. Cheng, ${ }^{36}$ D. J. Lange, ${ }^{36}$ M. C. Simani, ${ }^{36}$ D. M. Wright, ${ }^{36}$ A. J. Bevan, ${ }^{37}$ C. A. Chavez, ${ }^{37}$

J. P. Coleman, ${ }^{37}$ I. J. Forster, ${ }^{37}$ J. R. Fry, ${ }^{37}$ E. Gabathuler, ${ }^{37}$ R. Gamet, ${ }^{37}$ D. E. Hutcheroft,,${ }^{37}$ R. J. Parry, ${ }^{37}$ D. J. Payne ${ }^{37}$

C. Touramanis, ${ }^{37}$ C. M. Cormack, ${ }^{38}$ F. Di Lodovico, ${ }^{38}$ C. L. Brown, ${ }^{39}$ G. Cowan, ${ }^{39}$ R. L. Flack, ${ }^{39}$ H. U. Flaecher, ${ }^{39}$

M. G. Green,${ }^{39}$ P. S. Jackson, ${ }^{39}$ T. R. McMahon, ${ }^{39}$ S. Ricciardi, ${ }^{39}$ F. Salvatore, ${ }^{39}$ M. A. Winter,${ }^{39}$ D. Brown, ${ }^{40}$ C. L. Davis, ${ }^{40}$

J. Allison, ${ }^{41}$ N. R. Barlow, ${ }^{41}$ R. J. Barlow ${ }^{41}$ M. C. Hodgkinson, ${ }^{41}$ G. D. Lafferty, ${ }^{41}$ M. T. Naisbit, ${ }^{41}$ J. C. Williams, ${ }^{41}$

C. Chen, ${ }^{42}$ A. Farbin, ${ }^{42}$ W. D. Hulsbergen, ${ }^{42}$ A. Jawahery, ${ }^{42}$ D. Kovalskyi, ${ }^{42}$ C. K. Lae ${ }^{42}$ V. Lillard, ${ }^{42}$ D. A. Roberts, ${ }^{42}$

G. Blaylock ${ }^{43}$ C. Dallapiccola ${ }^{43}$ S. S. Hertzbach, ${ }^{43}$ R. Kofler, ${ }^{43}$ V. B. Koptchev, ${ }^{43}$ T. B. Moore, ${ }^{43}$ S. Saremi, ${ }^{43}$

H. Staengle ${ }^{43}$ S. Willocq, ${ }^{43}$ R. Cowan, ${ }^{44}$ K. Koeneke, ${ }^{44}$ G. Sciolla, ${ }^{44}$ S. J. Sekula ${ }^{44}$ F. Taylor, ${ }^{44}$ R. K. Yamamoto, ${ }^{44}$

P. M. Patel,${ }^{45}$ S. H. Robertson, ${ }^{45}$ A. Lazzaro, ${ }^{46}$ V. Lombardo, ${ }^{46}$ F. Palombo, ${ }^{46}$ J. M. Bauer,${ }^{47}$ L. Cremaldi, ${ }^{47}$

V. Eschenburg, ${ }^{47}$ R. Godang, ${ }^{47}$ R. Kroeger, ${ }^{47}$ J. Reidy, ${ }^{47}$ D. A. Sanders,${ }^{47}$ D. J. Summers, ${ }^{47}$ H. W. Zhao, ${ }^{47}$ S. Brunet, ${ }^{48}$ D. Côté, ${ }^{48}$ P. Taras,${ }^{48}$ H. Nicholson, ${ }^{49}$ N. Cavallo, ${ }^{50, *}$ F. Fabozzi, ${ }^{50, *}$ C. Gatto, ${ }^{50}$ L. Lista, ${ }^{50}$ D. Monorchio,${ }^{50}$ P. Paolucci, ${ }^{50}$ D. Piccolo,${ }^{50}$ C. Sciacca, ${ }^{50}$ M. Baak,${ }^{51}$ H. Bulten,${ }^{51}$ G. Raven, ${ }^{51}$ H. L. Snoek,${ }^{51}$ L. Wilden, ${ }^{51}$ C. P. Jessop, ${ }^{52}$ J. M. LoSecco, ${ }^{52}$ T. Allmendinger, ${ }^{33}$ G. Benelli, ${ }^{53}$ K. K. Gan, ${ }^{53}$ K. Honscheid, ${ }^{53}$ D. Hufnagel, ${ }^{53}$ H. Kagan, ${ }^{53}$ R. Kass, ${ }^{53}$ 
T. Pulliam, ${ }^{53}$ A. M. Rahimi, ${ }^{53}$ R. Ter-Antonyan, ${ }^{53}$ Q. K. Wong, ${ }^{53}$ J. Brau $^{54}$ R. Frey, ${ }^{54}$ O. Igonkina, ${ }^{54}$ M. Lu, ${ }^{54}$ C. T. Potter, ${ }^{54}$ N. B. Sinev, ${ }^{54}$ D. Strom, ${ }^{54}$ E. Torrence, ${ }^{54}$ F. Colecchia, ${ }^{55}$ A. Dorigo, ${ }^{55}$ F. Galeazzi, ${ }^{55}$ M. Margoni, ${ }^{55}$ M. Morandin, ${ }^{55}$ M. Posocco, ${ }^{55}$ M. Rotondo, ${ }^{55}$ F. Simonetto, ${ }^{55}$ R. Stroili, ${ }^{55}$ C. Voci, ${ }^{55}$ M. Benayoun, ${ }^{56}$ H. Briand, ${ }^{56}$ J. Chauveau, ${ }^{56}$ P. David, ${ }^{56}$ L. Del Buono, ${ }^{56}$ Ch. de la Vaissière, ${ }^{56}$ O. Hamon, ${ }^{56}$ M. J. J. John, ${ }^{56}$ Ph. Leruste, ${ }^{56}$ J. Malclès, ${ }^{56}$ J. Ocariz, ${ }^{56}$ L. Roos, ${ }^{56}$ G. Therin, ${ }^{56}$ P. K. Behera, ${ }^{57}$ L. Gladney, ${ }^{57}$ Q. H. Guo, ${ }^{57}$ J. Panetta, ${ }^{57}$ M. Biasini, ${ }^{58}$ R. Covarelli, ${ }^{58}$ M. Pioppi, ${ }^{58}$ C. Angelini, ${ }^{59}$ G. Batignani, ${ }^{59}$ S. Bettarini, ${ }^{59}$ M. Bondioli, ${ }^{59}$ F. Bucci, ${ }^{59}$ G. Calderini, ${ }^{59}$ M. Carpinelli, ${ }^{59}$ F. Forti, ${ }^{59}$ M. A. Giorgi, ${ }^{59}$ A. Lusiani, ${ }^{59}$ G. Marchiori, ${ }^{59}$ M. Morganti, ${ }^{59}$ N. Neri, ${ }^{59}$ E. Paoloni, ${ }^{59}$ M. Rama, ${ }^{59}$ G. Rizzo, ${ }^{59}$ G. Simi, ${ }^{59}$ J. Walsh, ${ }^{59}$ M. Haire, ${ }^{60}$ D. Judd, ${ }^{60}$ K. Paick, ${ }^{60}$ D. E. Wagoner, ${ }^{60}$ N. Danielson, ${ }^{61}$ P. Elmer, ${ }^{61}$ Y. P. Lau, ${ }^{61}$ C. Lu, ${ }^{61}$ V. Miftakov, ${ }^{61}$ J. Olsen, ${ }^{61}$ A. J. S. Smith, ${ }^{61}$ A. V. Telnov, ${ }^{61}$ F. Bellini, ${ }^{62}$ G. Cavoto, ${ }^{61,62}$ A. D' Orazio, ${ }^{62}$ E. Di Marco, ${ }^{62}$ R. Faccini, ${ }^{62}$ F. Ferrarotto, ${ }^{62}$ F. Ferroni, ${ }^{62}$ M. Gaspero, ${ }^{62}$ L. Li Gioi, ${ }^{62}$ M. A. Mazzoni, ${ }^{62}$ S. Morganti, ${ }^{62}$ G. Piredda, ${ }^{62}$ F. Polci, ${ }^{62}$ F. Safai Tehrani, ${ }^{62}$ C. Voena, ${ }^{62}$ S. Christ, ${ }^{63}$ H. Schröder, ${ }^{63}$ G. Wagner, ${ }^{63}$ R. Waldi, ${ }^{63}$ T. Adye, ${ }^{64}$ N. De Groot, ${ }^{64}$ B. Franek, ${ }^{64}$ G. P. Gopal, ${ }^{64}$ E. O. Olaiya, ${ }^{64}$ R. Aleksan, ${ }^{65}$ S. Emery, ${ }^{65}$ A. Gaidot, ${ }^{65}$ S. F. Ganzhur, ${ }^{65}$ P.-F. Giraud, ${ }^{65}$ G. Graziani, ${ }^{65}$ G. Hamel de Monchenault, ${ }^{65}$ W. Kozanecki, ${ }^{65}$ M. Legendre, ${ }^{65}$ G. W. London, ${ }^{65}$ B. Mayer, ${ }^{65}$ G. Vasseur, ${ }^{65}$ Ch. Yèche, ${ }^{65}$ M. Zito, ${ }^{65}$ M. V. Purohit, ${ }^{66}$ A. W. Weidemann, ${ }^{66}$ J. R. Wilson, ${ }^{66}$ F. X. Yumiceva, ${ }^{66}$ T. Abe, ${ }^{67}$ D. Aston, ${ }^{67}$ R. Bartoldus, ${ }^{67}$ N. Berger, ${ }^{67}$ A. M. Boyarski, ${ }^{67}$ O. L. Buchmueller, ${ }^{67}$ R. Claus, ${ }^{67}$ M. R. Convery, ${ }^{67}$ M. Cristinziani, ${ }^{67}$ G. De Nardo, ${ }^{67}$ J. C. Dingfelder, ${ }^{67}$ D. Dong, ${ }^{67}$ J. Dorfan, ${ }^{67}$ D. Dujmic, ${ }^{67}$ W. Dunwoodie, ${ }^{67}$ S. Fan, ${ }^{67}$ R. C. Field, ${ }^{67}$ T. Glanzman, ${ }^{67}$ S. J. Gowdy, ${ }^{67}$ T. Hadig, ${ }^{67}$ V. Halyo, ${ }^{67}$ C. Hast,${ }^{67}$ T. Hryn'ova, ${ }^{67}$ W. R. Innes, ${ }^{67}$ M. H. Kelsey, ${ }^{67}$ P. Kim, ${ }^{67}$ M. L. Kocian, ${ }^{67}$ D. W. G. S. Leith, ${ }^{67}$ J. Libby, ${ }^{67}$ S. Luitz, ${ }^{67}$ V. Luth, ${ }^{67}$ H. L. Lynch, ${ }^{67}$ H. Marsiske, ${ }^{67}$ R. Messner, ${ }^{67}$ D. R. Muller, ${ }^{67}$ C. P. O’Grady, ${ }^{67}$ V. E. Ozcan, ${ }^{67}$ A. Perazzo, ${ }^{67}$ M. Perl, ${ }^{67}$ B. N. Ratcliff, ${ }^{67}$ A. Roodman, ${ }^{67}$ A. A. Salnikov, ${ }^{67}$ R. H. Schindler, ${ }^{67}$ J. Schwiening, ${ }^{67}$ A. Snyder, ${ }^{67}$ A. Soha, ${ }^{67}$ J. Stelzer, ${ }^{67}$ J. Strube, ${ }^{54,67}$ D. Su, ${ }^{67}$ M. K. Sullivan, ${ }^{67}$ J. Va'vra, ${ }^{67}$ S. R. Wagner, ${ }^{67}$ M. Weaver, ${ }^{67}$ W. J. Wisniewski, ${ }^{67}$ M. Wittgen, ${ }^{67}$ D. H. Wright, ${ }^{67}$ A. K. Yarritu, ${ }^{67}$

C. C. Young, ${ }^{67}$ P. R. Burchat, ${ }^{68}$ A. J. Edwards, ${ }^{68}$ S. A. Majewski, ${ }^{68}$ B. A. Petersen, ${ }^{68}$ C. Roat, ${ }^{68}$ M. Ahmed, ${ }^{69}$ S. Ahmed, ${ }^{69}$ M. S. Alam, ${ }^{69}$ J. A. Ernst, ${ }^{69}$ M. A. Saeed, ${ }^{69}$ M. Saleem, ${ }^{69}$ F. R. Wappler, ${ }^{69}$ W. Bugg, ${ }^{70}$ M. Krishnamurthy, ${ }^{70}$

S. M. Spanier, ${ }^{70}$ R. Eckmann, ${ }^{71}$ H. Kim, ${ }^{71}$ J. L. Ritchie, ${ }^{71}$ A. Satpathy, ${ }^{71}$ R. F. Schwitters, ${ }^{71}$ J. M. Izen, $^{72}$ I. Kitayama, $^{72}$ X. C. Lou, ${ }^{72}$ S. Ye, ${ }^{72}$ F. Bianchi, ${ }^{73}$ M. Bona, ${ }^{73}$ F. Gallo, ${ }^{73}$ D. Gamba, ${ }^{73}$ L. Bosisio, ${ }^{74}$ C. Cartaro, ${ }^{74}$ F. Cossutti, ${ }^{74}$ G. Della Ricca, ${ }^{74}$ S. Dittongo, ${ }^{74}$ S. Grancagnolo, ${ }^{74}$ L. Lanceri, ${ }^{74}$ P. Poropat,${ }^{74}{ }^{\dagger}$ L. Vitale ${ }^{74}$ G. Vuagnin, ${ }^{74}$

F. Martinez-Vidal, ${ }^{2,75}$ R. S. Panvini, ${ }^{76}$ Sw. Banerjee, ${ }^{77}$ B. Bhuyan, ${ }^{77}$ C. M. Brown, ${ }^{77}$ D. Fortin, ${ }^{77}$ K. Hamano, ${ }^{77}$ P. D. Jackson, ${ }^{77}$ R. Kowalewski, ${ }^{77}$ J. M. Roney, ${ }^{77}$ R. J. Sobie, ${ }^{77}$ J. J. Back, ${ }^{78}$ P. F. Harrison, ${ }^{78}$ G. B. Mohanty, ${ }^{78}$ H. R. Band, ${ }^{79}$ X. Chen, ${ }^{79}$ B. Cheng, ${ }^{79}$ S. Dasu, ${ }^{79}$ M. Datta, ${ }^{79}$ A. M. Eichenbaum, ${ }^{79}$ K. T. Flood, ${ }^{79}$ M. Graham, ${ }^{79}$ J. J. Hollar, ${ }^{79}$ J. R. Johnson, ${ }^{79}$ P. E. Kutter, ${ }^{79}$ H. Li, ${ }^{79}$ R. Liu, ${ }^{79}$ A. Mihalyi, ${ }^{79}$ Y. Pan, ${ }^{79}$ R. Prepost, ${ }^{79}$ P. Tan, ${ }^{79}$ J. H. von Wimmersperg-Toeller, ${ }^{79}$ J. Wu, ${ }^{79}$ S. L. Wu, ${ }^{79}$ Z. Yu, ${ }^{79}$ M. G. Greene, ${ }^{80}$ and H. Neal ${ }^{80}$

(BABAR Collaboration)

\author{
${ }^{1}$ Laboratoire de Physique des Particules, F-74941 Annecy-le-Vieux, France \\ ${ }^{2}$ IFAE, Universitat Autonoma de Barcelona, E-08193 Bellaterra, Barcelona, Spain \\ ${ }^{3}$ Università di Bari, Dipartimento di Fisica and INFN, I-70126 Bari, Italy \\ ${ }^{4}$ Institute of High Energy Physics, Beijing 100039, China \\ ${ }^{5}$ University of Bergen, Inst. of Physics, N-5007 Bergen, Norway \\ ${ }^{6}$ Lawrence Berkeley National Laboratory and University of California, Berkeley, California 94720, USA \\ ${ }^{7}$ University of Birmingham, Birmingham, B15 2TT, United Kingdom \\ ${ }^{8}$ Ruhr Universität Bochum, Institut für Experimentalphysik 1, D-44780 Bochum, Germany \\ ${ }^{9}$ University of Bristol, Bristol BS8 1TL, United Kingdom \\ ${ }^{10}$ University of British Columbia, Vancouver, British Columbia V6T 1Z1, Canada \\ ${ }^{11}$ Brunel University, Uxbridge, Middlesex UB8 3PH, United Kingdom \\ ${ }^{12}$ Budker Institute of Nuclear Physics, Novosibirsk 630090, Russia \\ ${ }^{13}$ University of California at Irvine, Irvine, California 92697, USA \\ ${ }^{14}$ University of California at Los Angeles, Los Angeles, California 90024, USA \\ ${ }^{15}$ University of California at Riverside, Riverside, California 92521, USA \\ ${ }^{16}$ University of California at San Diego, La Jolla, California 92093, USA \\ ${ }^{17}$ University of California at Santa Barbara, Santa Barbara, California 93106, USA \\ ${ }^{18}$ University of California at Santa Cruz, Institute for Particle Physics, Santa Cruz, California 95064, USA \\ ${ }^{19}$ California Institute of Technology, Pasadena, California 91125, USA \\ ${ }^{20}$ University of Cincinnati, Cincinnati, Ohio 45221, USA
}




\author{
${ }^{21}$ University of Colorado, Boulder, Colorado 80309, USA \\ ${ }^{22}$ Colorado State University, Fort Collins, Colorado 80523, USA \\ ${ }^{23}$ Universität Dortmund, Institut fur Physik, D-44221 Dortmund, Germany \\ ${ }^{24}$ Technische Universität Dresden, Institut für Kernund Teilchenphysik, D-01062 Dresden, Germany \\ ${ }^{25}$ Ecole Polytechnique, LLR, F-91128 Palaiseau, France \\ ${ }^{26}$ University of Edinburgh, Edinburgh EH9 3JZ, United Kingdom \\ ${ }^{27}$ Università di Ferrara, Dipartimento di Fisica and INFN, I-44100 Ferrara, Italy \\ ${ }^{28}$ Laboratori Nazionali di Frascati dell', INFN, I-00044 Frascati, Italy \\ ${ }^{29}$ Università di Genova, Dipartimento di Fisica and INFN, I-16146 Genova, Italy \\ ${ }^{30}$ Harvard University, Cambridge, Massachusetts 02138, USA \\ ${ }^{31}$ Universität Heidelberg, Physikalisches Institut, Philosophenweg 12, D-69120 Heidelberg, Germany \\ ${ }^{32}$ Imperial College London, London, SW7 2AZ, United Kingdom \\ ${ }^{33}$ University of Iowa, Iowa City, Iowa 52242, USA \\ ${ }^{34}$ Iowa State University, Ames, Iowa 50011-3160, USA \\ ${ }^{35}$ Laboratoire de l'Accélérateur Linéaire, F-91898 Orsay, France \\ ${ }^{36}$ Lawrence Livermore National Laboratory, Livermore, California 94550, USA \\ ${ }^{37}$ University of Liverpool, Liverpool L69 72E, United Kingdom \\ ${ }^{38}$ Queen Mary, University of London, E1 4NS, United Kingdom \\ ${ }^{39}$ University of London, Royal Holloway and Bedford New College, Egham, Surrey TW20 OEX, United Kingdom \\ ${ }^{40}$ University of Louisville, Louisville, Kentucky 40292, USA \\ ${ }^{41}$ University of Manchester, Manchester M13 9PL, United Kingdom \\ ${ }^{42}$ University of Maryland, College Park, Maryland 20742, USA \\ ${ }^{43}$ University of Massachusetts, Amherst, Massachusetts 01003, USA \\ ${ }^{44}$ Massachusetts Institute of Technology, Laboratory for Nuclear Science, Cambridge, Massachusetts 02139, USA \\ ${ }^{45}$ Mc Gill University, Montréal, Quebec, Canada H3A $2 T 8$ \\ ${ }^{46}$ Università di Milano, Dipartimento di Fisica and INFN, I-20133 Milano, Italy \\ ${ }^{47}$ University of Mississippi, University, Mississippi 38677, USA \\ ${ }^{48}$ Université de Montréal, Laboratoire René J. A. Lévesque, Montréal, Quebec H3C 3J7, Canada \\ ${ }^{49}$ Mount Holyoke College, South Hadley, Massachusetts 01075, USA \\ ${ }^{50}$ Università di Napoli Federico II, Dipartimento di Scienze Fisiche and INFN, I-80126, Napoli, Italy \\ ${ }^{51}$ NIKHEF, National Institute for Nuclear Physics and High Energy Physics, NL-1009 DB Amsterdam, The Netherlands \\ ${ }^{52}$ University of Notre Dame, Notre Dame, Indiana 46556, USA \\ ${ }^{53}$ Ohio State University, Columbus, Ohio 43210, USA \\ ${ }^{54}$ University of Oregon, Eugene, Oregon 97403, USA \\ ${ }^{55}$ Università di Padova, Dipartimento di Fisica and INFN, I-35131 Padova, Italy \\ ${ }^{56}$ Universités Paris VI et VII, Laboratoire de Physique Nucléaire et de Hautes Energies, F-7 5252 Paris, France \\ ${ }^{57}$ University of Pennsylvania, Philadelphia, Pennsylvania 19104, USA \\ ${ }^{58}$ Università di Perugia, Dipartimento di Fisica and INFN, I-06100 Perugia, Italy \\ ${ }^{59}$ Università di Pisa, Dipartimento di Fisica, Scuola Normale Superiore and INFN, I-56127 Pisa, Italy \\ ${ }^{60}$ Prairie View A\&M University, Prairie View, Texas 77446, USA \\ ${ }^{61}$ Princeton University, Princeton, New Jersey 08544, USA \\ ${ }^{62}$ Università di Roma La Sapienza, Dipartimento di Fisica and INFN, I-00185 Roma, Italy \\ ${ }^{63}$ Universität Rostock, D-18051 Rostock, Germany \\ ${ }^{64}$ Rutherford Appleton Laboratory, Chilton, Didcot, Oxon, OX11 OQX, United Kingdom \\ ${ }^{65}$ DSM/Dapnia, CEA/Saclay, F-91191 Gif-sur-Yvette, France \\ ${ }^{66}$ University of South Carolina, Columbia, South Carolina 29208, USA \\ ${ }^{67}$ Stanford Linear Accelerator Center, Stanford, California 94309, USA \\ ${ }^{68}$ Stanford University, Stanford, California 94305-4060, USA \\ ${ }^{69}$ State University of New York, Albany, New York 12222, USA \\ ${ }^{70}$ University of Tennessee, Knoxville, Tennessee 37996, USA \\ ${ }^{71}$ University of Texas at Austin, Austin, Texas 78712, USA \\ ${ }^{72}$ University of Texas at Dallas, Richardson, Texas 75083, USA \\ ${ }^{73}$ Università di Torino, Dipartimento di Fisica Sperimentale and INFN, I-10125 Torino, Italy \\ ${ }^{74}$ Università di Trieste, Dipartimento di Fisica and INFN, I-34127 Trieste, Italy \\ ${ }^{75}$ IFIC, Universitat de Valencia-CSIC, E-46071 Valencia, Spain \\ ${ }^{76}$ Vanderbilt University, Nashville, Tennessee 37235, USA \\ ${ }^{77}$ University of Victoria, Victoria, British Columbia V8W 3P6, Canada \\ ${ }^{78}$ Department of Physics, University of Warwick, Coventry CV4 7AL, United Kingdom \\ ${ }^{79}$ University of Wisconsin, Madison, Wisconsin 53706, USA \\ ${ }^{80}$ Yale University, New Haven, Connecticut 06511, USA \\ (Received 23 December 2004; published 8 April 2005)
}


We search for the decay $B^{0} \rightarrow \rho^{0} \rho^{0}$ in a data sample of about $227 \times 10^{6} \Upsilon(4 S) \rightarrow B \bar{B}$ decays collected with the BABAR detector at the PEP-II asymmetric-energy $e^{+} e^{-}$collider at SLAC. We find no significant signal and set an upper limit of $1.1 \times 10^{-6}$ at $90 \%$ C.L. on the branching fraction. As a result, the uncertainty due to penguin contributions on the Cabibbo-Kobayashi-Maskawa unitarity angle $\alpha$ measured in $B \rightarrow \rho \rho$ decays is decreased to $11^{\circ}$ at $68 \%$ C.L.

DOI: 10.1103/PhysRevLett.94.131801

PACS numbers: 13.25.Hw, 11.30.Er, 12.15.Hh

Measurements of $C P$-violating asymmetries in the $B^{0} \bar{B}^{0}$ system provide tests of the standard model by overconstraining the Cabibbo-Kobayashi-Maskawa (CKM) quark-mixing matrix [1] through the measurement of the unitarity angles. Measuring the time-dependent $C P$ asymmetry in a neutral- $B$-meson decay to a $C P$ eigenstate dominated by the tree-level amplitude $b \rightarrow u \bar{u} d$ gives an approximation $\alpha_{\text {eff }}$ to the CKM unitarity angle $\alpha \equiv$ $\arg \left[-V_{t d} V_{t b}^{*} / V_{u d} V_{u b}^{*}\right]$. The correction $\Delta \alpha=\alpha-\alpha_{\text {eff }}$, which accounts for the effects of penguin-amplitude contributions as an additional decay mechanism, can be extracted from an isospin analysis of the branching fractions of the $B$ decays into the full set of isospin-related channels [2].

Measurements of branching fractions and timedependent $C P$ asymmetries in $B \rightarrow \pi \pi, \rho \pi$, and $\rho \rho$ have already provided information on $\alpha$. Because the branching fraction for $B^{0} \rightarrow \pi^{0} \pi^{0}$ is comparable to that for $B^{+} \rightarrow \pi^{+} \pi^{0}$ and $B^{0} \rightarrow \pi^{+} \pi^{-}$, the limit on the correction is weak: $\left|\Delta \alpha_{\pi \pi}\right|<35^{\circ}$ at $90 \%$ confidence level (C.L.) [3]. (Charge conjugate $B$ decay modes are implied in this Letter.) In contrast, the $\rho^{0} \rho^{0}$ channel has a much smaller branching fraction than the channels with charged $\rho$ 's [4-7]. As a consequence, it is possible to set a tighter limit on $\Delta \alpha_{\rho \rho}[2,6,8]$. This makes the $\rho \rho$ system particularly effective for measuring $\alpha$ in a model-independent way.

In $B \rightarrow \rho \rho$ decays the final state is a superposition of $C P$-odd and $C P$-even states, and an isospin-triangle relation [2] holds for each of the three helicity amplitudes, which can be separated through an angular analysis. The measured polarizations in $B^{+} \rightarrow \rho^{+} \rho^{0}[4,5]$ and $B^{0} \rightarrow$ $\rho^{+} \rho^{-}[6,7]$ modes indicate that the $\rho^{\prime}$ 's are nearly entirely longitudinally polarized. The current best limit on the $B^{0} \rightarrow \rho^{0} \rho^{0}$ branching fraction was obtained by $B A B A R$ with a sample of $89 \times 10^{6} \Upsilon(4 S) \rightarrow B \bar{B}$ decays [4].

In this Letter we present improved constraints on the $B^{0} \rightarrow \rho^{0} \rho^{0}$ branching fraction and the penguin contribution to the measurement of the unitarity angle $\alpha$. These results are based on data collected with the $B A B A R$ detector [9] at the PEP-II asymmetric-energy $e^{+} e^{-}$collider [10] located at the Stanford Linear Accelerator Center. A sample of $226.6 \pm 2.5$ million $B \bar{B}$ pairs, corresponding to an integrated luminosity of approximately $205 \mathrm{fb}^{-1}$, was recorded at the $\mathrm{Y}(4 S)$ resonance with the center-of-mass (c.m.) energy $\sqrt{s}=10.58 \mathrm{GeV}$. We use a sample of $16 \mathrm{fb}^{-1}$ taken $40 \mathrm{MeV}$ below the $\mathrm{Y}(4 S)$ resonance to study background contributions from $e^{+} e^{-} \rightarrow q \bar{q}(q=u, d, s$, or $c$ ) continuum events.

To reconstruct $B^{0} \rightarrow \rho^{0} \rho^{0} \rightarrow\left(\pi^{+} \pi^{-}\right)\left(\pi^{+} \pi^{-}\right)$candidates, we select four charged tracks that are consistent with originating from a single vertex near the $e^{+} e^{-}$interaction point. Particle identification is provided by measurements of the energy loss in the silicon vertex tracker and the drift chamber and by the Cherenkov angle in an internally reflecting ring-imaging Cherenkov detector [9].

The angular distribution of the $B^{0} \rightarrow \rho^{0} \rho^{0}$ decay products can be expressed as a function of the helicity angles $\left(\theta_{1}, \theta_{2}, \phi\right)$, which are defined by the directions of the twobody $\rho^{0}$ decay axes and the direction opposite the $B$ in the $\rho^{0}$ rest systems, as shown in Fig. 1 . Since the detector acceptance does not depend on $\phi$, the resulting angular distribution $d^{2} \Gamma /\left(\Gamma d \cos \theta_{1} d \cos \theta_{2}\right)$ is

$$
\frac{9}{4}\left\{\frac{1}{4}\left(1-f_{L}\right) \sin ^{2} \theta_{1} \sin ^{2} \theta_{2}+f_{L} \cos ^{2} \theta_{1} \cos ^{2} \theta_{2}\right\},
$$

where $f_{L}=\left|A_{0}\right|^{2} /\left(\Sigma\left|A_{\lambda}\right|^{2}\right)$ is the longitudinal polarization fraction and $A_{\lambda=-1,0,+1}$ are the helicity amplitudes.

The identification of signal $B$ candidates is based on two kinematic variables: the beam-energy-substituted mass, $m_{\mathrm{ES}}=\left[\left(s / 2+\mathbf{p}_{i} \cdot \mathbf{p}_{B}\right)^{2} / E_{i}^{2}-\mathbf{p}_{B}^{2}\right]^{1 / 2}$, where the initial total $e^{+} e^{-}$four momentum $\left(E_{i}, \mathbf{p}_{i}\right)$ and the $B$ momentum $\mathbf{p}_{\mathbf{B}}$ are defined in the laboratory frame; and the difference between the reconstructed $B$ energy in the c.m. frame and its known value $\Delta E=E_{B}^{\mathrm{cm}}-\sqrt{s} / 2$. The signal $m_{\mathrm{ES}}$ and $\Delta E$ resolutions are $2.6 \mathrm{MeV} / \mathrm{c}^{2}$ and $20 \mathrm{MeV}$, respectively. The selection requirements for $m_{\mathrm{ES}}, \Delta E$, the two $\pi^{+} \pi^{-}$invariant masses $m_{1,2}$, and the helicity angles are the following: $5.24<m_{\mathrm{ES}}<5.29 \mathrm{GeV} / \mathrm{c}^{2}, \quad|\Delta E|<$ $85 \mathrm{MeV}, \quad 0.55<m_{1,2}<1.00 \mathrm{GeV} / \mathrm{c}^{2}$, and $\left|\cos \theta_{1,2}\right|<$ 0.99 . The last requirement removes a region with low reconstruction efficiency.

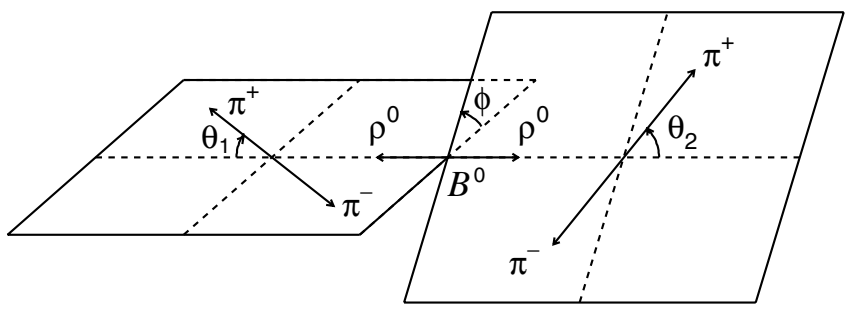

FIG. 1. Definition of helicity angles $\theta_{1}, \theta_{2}$, and $\phi$ for the decay $B^{0} \rightarrow \rho^{0} \rho^{0}$. The $\rho^{0}$ final states are shown in the $\rho^{0}$ rest frames. 
To reject the dominant continuum background we require $\left|\cos \theta_{T}\right|<0.8$, where $\theta_{T}$ is the angle between the $B$-candidate thrust axis and that of the remaining tracks and neutral clusters in the event, calculated in the c.m. frame. We also use as discriminating variables the polar angles of the $B$ momentum vector and the $B$-candidate thrust axis with respect to the beam axis in the c.m. frame, and the two Legendre moments $L_{0}$ and $L_{2}$ of the energy flow around the $B$-candidate thrust axis [11]. These variables are combined in a neural network, the output of which is transformed into a variable $\mathcal{E}$ for which the signal and background distributions are approximately Gaussian.

We veto the background mode $B^{0} \rightarrow D^{-} \pi^{+} \rightarrow$ $h^{+} \pi^{-} \pi^{-} \pi^{+}$, where $h^{+}$refers to a pion or kaon. We require the invariant mass of the three-particle combination that excludes the highest-momentum track in the candidate $B$ rest frame to be inconsistent with being the $D$-meson mass $\left(\left|m_{h \pi \pi}-m_{D}\right|>13 \mathrm{MeV} / \mathrm{c}^{2}\right)$. After application of all selection criteria, $N_{\text {cand }}=35740$ events are retained, most of which are background events. On average each selected event has 1.05 candidates. When more than one candidate is present in the same event, one candidate is selected randomly.

The signal selection efficiency determined from Monte Carlo (MC) [12] simulation is $27 \%$ or $32 \%$ for longitudinally or transversely polarized events, respectively. MC simulation shows that $22 \%$ of longitudinally and $8 \%$ of transversely polarized signal events are misreconstructed with one or more tracks not originating from the $B^{0} \rightarrow \rho^{0} \rho^{0}$ decay. These are mostly due to combinatorial background from low-momentum tracks from the other $B$. We treat these as part of the signal.

Further background separation is achieved by the use of multivariate $B$-flavor-tagging algorithms trained to identify primary leptons, kaons, soft pions and high-momentum charged particles from the other $B$ in the event [13]. The discrimination power arises from the difference between the tagging efficiencies for signal and background in five tagging categories $c_{\text {tag. }}$.

We use an unbinned extended maximum likelihood fit to extract the $B^{0} \rightarrow \rho^{0} \rho^{0}$ event yield. The likelihood function is

$$
\mathcal{L}=\exp \left(-\sum_{k} n_{k}\right) \prod_{i=1}^{N_{\text {cand }}}\left(\sum_{j} n_{j} \mathcal{P}_{j}\left(\vec{x}_{i}\right)\right),
$$

where $n_{j}$ is the number of events for each hypothesis $j$ (signal, continuum, and six $B$-background classes), and $\mathcal{P}_{j}\left(\vec{x}_{i}\right)$ is the corresponding probability density function (PDF), evaluated with the variables $\vec{x}_{i}=\left\{m_{\mathrm{ES}}, \Delta E, \mathcal{E}\right.$, $\left.m_{1}, m_{2}, \cos \theta_{1}, \cos \theta_{2}, c_{\text {tag }}\right\}$ of the $i$ th event.

We use MC-simulated events to study the background from other $B$ decays. The charmless modes are grouped into five classes with similar kinematic and topological properties: $B^{0} \rightarrow a_{1}^{ \pm} \pi^{\mp} ; B^{0} \rightarrow \rho^{0} K^{* 0} ; B^{+} \rightarrow \rho^{+} \rho^{0} ;$ a combination of $B \rightarrow \rho \pi$ and $B^{0} \rightarrow \rho^{+} \rho^{-}$; and $B$ decays to other charmless modes not included explicitly. One additional class accounts for the remaining neutral and charged $B$ decays to charm modes. The number of events in each class $n_{j}$ is left free in the fit with the exception of three classes where $n_{j}$ is fixed either to the expectations from independent measurements (78 \pm 20 events of $B^{+} \rightarrow$ $\rho^{+} \rho^{0}$, and $48 \pm 8$ events of $B \rightarrow \rho \pi$ and $B^{0} \rightarrow \rho^{+} \rho^{-}$) or to the extrapolation from the flavor-SU(3)-related $B$-decay modes [4] ( $25 \pm 18$ events of $\left.B^{0} \rightarrow \rho^{0} K^{* 0}\right)$.

Since the correlations among the variables are found to be small, we take each $\mathcal{P}_{j}$ as the product of the PDFs for the separate variables. Exceptions are the correlation between the two helicity angles in signal, and mass-helicity correlations in backgrounds and misrecontructed signal, taken into account as discussed below.

We use double-Gaussian functions to parameterize the $m_{\mathrm{ES}}$ and $\Delta E$ PDFs for the signal, and a relativistic $P$-wave Breit-Wigner (BW) formula convoluted with a Gaussian resolution function for the resonance masses. The angular distribution for signal, expressed as a function of the longitudinal polarization in Eq. (1), is multiplied by a detector acceptance function $G\left(\cos \theta_{1}, \cos \theta_{2}\right)$, obtained with MC simulation. The distributions of misreconstructed signal events are parameterized with empirical shapes in a fashion similar to that used for $B$ background, as described below. The $\mathcal{E}$ variable is described by two asymmetric Gaussian functions with different parameters for signal and background distributions.

The $m_{\mathrm{ES}}$ distribution of the continuum background is described with the ARGUS parameterization [14]. The $\Delta E$ and resonance mass $m_{1,2}$ PDFs are parameterized with lowdegree polynomials. The parameterization of the $m_{1}$ and $m_{2}$ distributions includes a BW resonant component to account for the real $\rho^{0}$ resonances in the continuum background, which are assumed to be unpolarized and thus to have a flat distribution in $\cos \theta_{1,2}$. The $\cos \theta_{1,2}$ distribution of the continuum background excluding the real resonances is parameterized with a second-degree polynomial and an exponential function to allow for the increased fraction of combinatorial $\pi^{+} \pi^{-}$candidates with lowmomentum pions near $\left|\cos \theta_{1,2}\right|=1$. This parameterization depends on the $\rho$ candidate's mass.

The PDFs for exclusive nonsignal $B$ decay modes are generally modeled with empirical nonparametric distributions [15]. However, analytical distributions are used for the variables that have distributions identical to those for signal, such as $m_{\mathrm{ES}}$ when all four tracks come from the same $B$, or $\pi^{+} \pi^{-}$invariant mass $m_{1,2}$ when both tracks come from a $\rho^{0}$ meson. The two $\rho^{0}$ candidates of some exclusive nonsignal modes can have very different mass and helicity distributions. This occurs when one of the two $\rho^{0}$ candidates is real (e.g., $\rho^{+} \rho^{0}, \rho^{0} K^{* 0}$ ) or when one of the two $\rho^{0}$ candidates contains a high-momentum pion $\left(a_{1} \pi\right)$. In such cases, we use a four-variable correlated mass-helicity PDF. 
The signal and $B$-background PDF parameters are extracted from MC simulation while the continuum background PDF parameters are obtained from data in $m_{\mathrm{ES}}$ and $\Delta E$ sidebands. The MC parameters of $m_{\mathrm{ES}}, \Delta E$, and $\mathcal{E}$ are adjusted by comparing data and $\mathrm{MC}$ in calibration channels with similar kinematics and topology, such as $B^{0} \rightarrow$ $D^{-} \pi^{+}$with $D^{-} \rightarrow K^{+} \pi^{-} \pi^{-}$. Finally, the $B$-flavor tagging PDFs for all decay modes are the normalized discrete $c_{\text {tag }}$ distributions of tagging categories. Large samples of fully reconstructed $B$-meson decays are used to obtain the $B$-tagging efficiencies for signal $B$ decays and to study systematic uncertainties in the MC values of $B$-tagging efficiencies for the $B$ backgrounds.

Table I shows the results of the fit. No significant signal yield is observed. We obtain an upper limit by integrating the normalized likelihood distribution over the positive values of the branching fraction. The value of $f_{L}$ is fixed to 1 in the fit, as this assumption has been shown to give the most conservative upper limit and it approximates the values obtained in the $B \rightarrow \rho \rho$ decays dominated by the tree-level amplitude. The statistical significance is taken as the square root of the change in $-2 \ln \mathcal{L}$ when the number of signal events is constrained to zero in the likelihood fit. In Fig. 2 we show the projections of the fit results onto $m_{\mathrm{ES}}$ and $\Delta E$.

Systematic errors in the fit originate from uncertainties in the PDF parameterizations, which arise from the limited number of events in the sideband data and signal control samples. The PDF parameters are varied by their respective uncertainties to derive the corresponding systematic errors (6.0 events). The event yields from the $B$-background modes fixed in the fit are varied according to the uncertainties in the measured or estimated branching fractions. This results in a systematic error on the signal yield of 5.8 events. We also assign a systematic error of 3.0 events to cover a possible fit bias, evaluated with $\mathrm{MC}$ experiments.

We estimate the systematic uncertainty due to signal- $a_{1}^{ \pm} \pi^{\mp}$ interference using a simulation study in which the decay amplitudes for $B^{0} \rightarrow \rho^{0} \rho^{0}$ are generated according to this measurement and those for $B^{0} \rightarrow a_{1}^{ \pm} \pi^{\mp}$ correspond to a branching fraction of $4 \times 10^{-5}$ [16]. The relative phases between these are modeled with BW am-

TABLE I. Summary of results: signal yield $\left(n_{\text {sig }}\right)$, selection efficiency (Eff), branching fraction $(\mathcal{B})$, branching fraction upper limit (UL) at 90\% C.L., and significance (including systematic uncertainties). The assumption $f_{L}=1$ is used. The systematic errors are quoted last.

\begin{tabular}{lc}
\hline \hline Quantity & \multicolumn{1}{c}{ Value } \\
\hline$n_{\text {sig }}($ events $)$ & $33_{-20}^{+22} \pm 12$ \\
Eff $(\%)$ & $27.1 \pm 1.3$ \\
$\mathcal{B}\left(\times 10^{-6}\right)$ & $0.54_{-0.32}^{+0.36} \pm 0.19$ \\
UL $\left(\times 10^{-6}\right)$ & 1.1 \\
Significance $(\sigma)$ & 1.6 \\
\hline \hline
\end{tabular}

plitudes for all $\rho \rightarrow \pi \pi$ and $a_{1} \rightarrow \rho \pi$ combinations, with additional constants. The values of the constants and the $a_{1}^{ \pm} \pi^{\mp} C P$ asymmetries are varied over the allowed ranges. We take the rms variation of the average signal yield (7.5 events) as a systematic uncertainty.

Uncertainties in the reconstruction efficiency arise from track finding (3\%), particle identification (2\%), and other selection requirements, such as on vertex probability $(2 \%)$, track multiplicity (1\%), and thrust angle (1\%).

Our measurement confirms the small value of the $B^{0} \rightarrow$ $\rho^{0} \rho^{0}$ branching fraction with the statistical uncertainty improved by approximately a factor of 2 over our previous result [4]. Since the tree contribution to the $B^{0} \rightarrow \rho^{0} \rho^{0}$ decay is color suppressed, the decay rate is sensitive to the penguin amplitude. Thus, this mode has important implications for constraining the uncertainty due to penguin contributions in the measurement of the CKM unitarity angle $\alpha$ with $B \rightarrow \rho \rho$ decays.

In the isospin analysis [2], we minimize a $\chi^{2}$ that includes the measured quantities expressed as the lengths of the sides of the isospin triangles. We use the measured branching fractions and fractions of longitudinal polarization of the $B^{+} \rightarrow \rho^{+} \rho^{0}[4,5]$ and $B^{0} \rightarrow \rho^{+} \rho^{-}[6,7]$ decays, the $C P$-violating parameters $S_{L}^{+-}$and $C_{L}^{+-}$obtained from the time evolution of the longitudinally polarized $B^{0} \rightarrow \rho^{+} \rho^{-}$decay [7], and the branching fraction of $B^{0} \rightarrow$ $\rho^{0} \rho^{0}$ from this analysis. We neglect isospin-breaking effects, nonresonant, and $I=1$ isospin contributions [8].

With the $B^{0} \rightarrow \rho^{0} \rho^{0}$ measurement we improve the constraint on $\alpha$ due to the penguin contribution and obtain a $68 \%(90 \%)$ C.L. limit on $\Delta \alpha_{\rho \rho}=\alpha-\alpha_{\text {eff }}$ of $\pm 11^{\circ}$ $\left( \pm 14^{\circ}\right)$. Figure 3 shows the $\Delta \chi^{2}$ on $\Delta \alpha_{\rho \rho}$. Since the central value from Fig. 3 is $\Delta \alpha_{\rho \rho}=0$, the central value of $\alpha$ obtained from the isospin analysis is the same as $\alpha_{\text {eff }}$, which is constrained by the relation $\sin \left(2 \alpha_{\text {eff }}\right)=$ $S_{L}^{+-} /\left(1-C_{L}^{+-2}\right)^{1 / 2}$ and is measured with the $B^{0} \rightarrow \rho^{+} \rho^{-}$
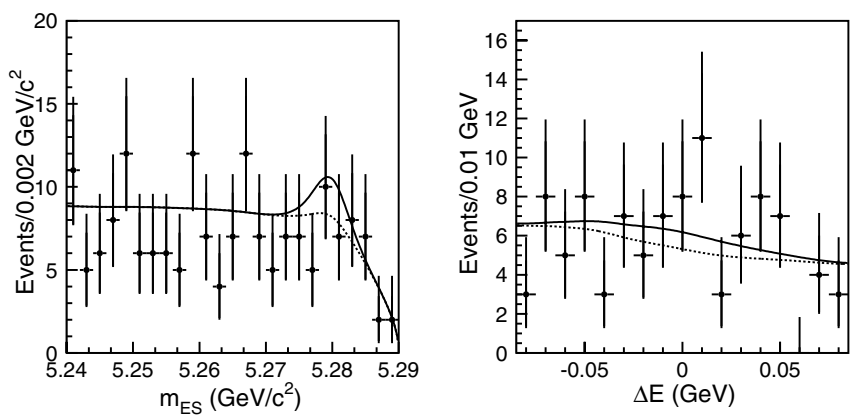

FIG. 2. Projections of the multidimensional fit onto $m_{\mathrm{ES}}$ and $\Delta E$ after a requirement on the signal-to-background probability ratio $\mathcal{P}_{\text {sig }} / \mathcal{P}_{\text {bkg }}$ with the plotted variable excluded. This requirement maximizes the fraction of signal events in the sample, which contains $22.5 \%$ and $23.9 \%$ of signal, and less than $0.5 \%$ and $0.2 \%$ of continuum background, respectively. The histogram shows the data and the solid (dashed) line shows the full (background only) PDF projection. 


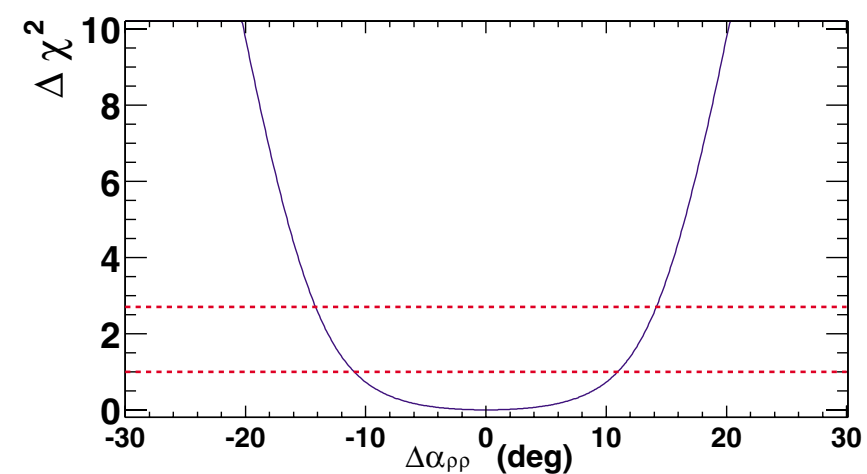

FIG. 3 (color online). $\Delta \chi^{2}$ on $\Delta \alpha_{\rho \rho}$ obtained from the isospin analysis discussed in the text. The dashed lines at $\Delta \chi^{2}=1$ and $\Delta \chi^{2}=2.7$ are taken for the $1 \sigma(68 \%)$ and $1.64 \sigma(90 \%)$ interval estimates.

decay [7] to be $\alpha_{\text {eff }}=\left[102_{-12}^{+16}(\mathrm{stat})_{-4}^{+5} \text { (syst) }\right]^{\circ}$ at $68 \%$ C.L., where the solution closest to the CKM best fit central value $[17,18]$ is chosen. For this solution we obtain the penguinto-tree [19] $B^{0} \rightarrow \rho^{+} \rho^{-}$amplitude ratio $r_{\rho \rho}^{+-}=0.07_{-0.07}^{+0.14}$, where we adopt the $C$ convention defined in Ref. [17], and where the corresponding CKM factors are included in the ratio.

The error due to the penguin contribution may become the dominant uncertainty in the measurement of $\alpha$ using $B \rightarrow \rho \rho$ decays. However, if $B^{0} \rightarrow \rho^{0} \rho^{0}$ decays are observed, time-dependent and angular analyses will allow us to measure the $C P$ parameters $S_{L}^{00}$ and $C_{L}^{00}$, analogous to $S_{L}^{+-}$and $C_{L}^{+-}$, resolving ambiguities inherent to isospintriangle orientations.

In summary, we have improved the precision on the measurement of the $B^{0} \rightarrow \rho^{0} \rho^{0}$ branching fraction by approximately a factor of 2 . The limit on this branching fraction relative to those for $B^{+} \rightarrow \rho^{0} \rho^{+}$and $B^{0} \rightarrow \rho^{+} \rho^{-}$ provides a tight constraint on the penguin uncertainty in the determination of the CKM unitarity angle $\alpha$. The results summarized in Table I supersede our previous measurement [4].

We are grateful for the excellent luminosity and machine conditions provided by our PEP-II colleagues, and for the substantial dedicated effort from the computing organizations that support $B A B A R$. The collaborating institutions wish to thank SLAC for its support and kind hospitality. This work is supported by DOE and NSF (USA), NSERC
(Canada), IHEP (China), CEA and CNRS-IN2P3 (France), BMBF and DFG (Germany), INFN (Italy), FOM (The Netherlands), NFR (Norway), MIST (Russia), and PPARC (United Kingdom). Individuals have received support from CONACyT (Mexico), A. P. Sloan Foundation, Research Corporation, and Alexander von Humboldt Foundation.

*Also at Università della Basilicata, Potenza, Italy ${ }^{\dagger}$ Deceased

[1] M. Kobayashi and T. Maskawa, Prog. Theor. Phys. 49, 652 (1973); N. Cabibbo, Phys. Rev. Lett. 10, 531 (1963).

[2] M. Gronau and D. London, Phys. Rev. Lett. 65, 3381 (1990).

[3] BABAR Collaboration, B. Aubert et al., hep-ex/0412037, [Phys. Rev. Lett. (to be published)].

[4] BABAR Collaboration, B. Aubert et al., Phys. Rev. Lett. 91, 171802 (2003).

[5] Belle Collaboration, J. Zhang et al., Phys. Rev. Lett. 91, 221801 (2003).

[6] BABAR Collaboration, B. Aubert et al., Phys. Rev. D 69, 031102 (2004).

[7] BABAR Collaboration, B. Aubert et al., Phys. Rev. Lett. 93, 231801 (2004).

[8] A. F. Falk et al., Phys. Rev. D 69, 011502 (2004).

[9] BABAR Collaboration, B. Aubert et al., Nucl. Instrum. Methods Phys. Res., Sect. A 479, 1 (2002).

[10] PEP-II Conceptual Design Report, SLAC-R-418 (1993).

[11] BABAR Collaboration, B. Aubert et al., Phys. Rev. Lett. 89, 281802 (2002); , Phys. Rev. D 70, 032006 (2004).

[12] The BABAR detector Monte Carlo simulation is based on GEANT4: S. Agostinelli et al., Nucl. Instrum. Methods Phys. Res., Sect. A 506, 250 (2003).

[13] BABAR Collaboration, B. Aubert et al., Phys. Rev. Lett. 89, 201802 (2002).

[14] ARGUS Collaboration, H. Albrecht et al., Z. Phys. C 48, 543 (1990).

[15] K. S. Cranmer, Comput. Phys. Commun. 136, 198 (2001).

[16] BABAR Collaboration, B. Aubert et al., hep-ex/0408021.

[17] J. Charles et al., hep-ph/0406184, to be published [Eur. Phys. J. C (to be published)].

[18] M. Bona et al., hep-ph/0408079.

[19] In the $C$ convention [17], the tree amplitude includes the contribution from the difference of the $u$ and the $c$ penguin-type amplitudes, while the penguin amplitude is the difference between the $t$ and $c$ penguin amplitudes. 